\title{
Darstellung von Aryl- und Heteroaryltrimethylsilanen durch ,in-situ“-Grignard-Synthese
}

\author{
Franz Effenberger $\left.\left.{ }^{*}\right) * *\right)$ und Dieter Häbich ${ }^{1)}$
}

Institut für Organische Chemie der Universität Stuttgart, Pfaffenwaldring 55, D-7000 Stuttgart 80

Eingegangen am 23. Dezember 1978

Aryl- und Heteroarylhalogenide reagieren beim Erwärmen mit Magnesium und Chlortrimethylsilan in Hexamethylphoshorsäuretriamid in guten Ausbeuten zu Aryl- und Heteroaryltrimethylsilanen. Die Vorteile dieser ,in-situ“-Grignard-Synthese gegenüber anderen Verfahren werden beschrieben, Anwendungsbreite und Grenzen dieser Methode bei verschieden substituierten Aromaten sowie unterschiedlichen Heterocyclen untersucht.

\section{Preparation of Aryl- and Heteroaryl-trimethylsilanes - Scope of "in situ" Grignard Synthesis}

Aryl and heteroaryl halides react upon heating with magnesium and chlorotrimethylsilane in hexamethyl phosphoric triamide to give aryl and heteroaryl trimethylsilanes in good yields. The advantage of the "in situ" Grignard synthesis over other methods is demonstrated. The scope and limit of this synthesis is investigated for variously substituted aryl compounds and various heterocyclic systems.

Desilylierungsreaktionen von Aryl- und Heteroaryltrimethylsilanen mit Elektrophilen bieten gegenüber konventionellen Aromatensubstitutionen häufig präparative Vorteile, so z. B. bei der Darstellung von schwer zugänglichen Isomeren oder bei der Umsetzung von säureempfindlichen Verbindungen ${ }^{2}$.

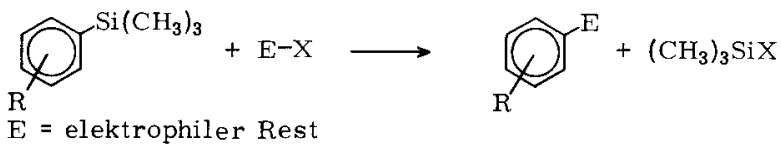

Voraussetzung einer breiten Anwendung solcher Desilylierungsreaktionen ist jedoch ein bequemer, möglichst allgemein anwendbarer Zugang zu den erforderlichen Arylsilanen. Gewöhnlich werden diese Substanzen durch Umsetzung der entsprechenden Grignard- ${ }^{3)}$, Lithium- ${ }^{4)}$ oder Natrium-Verbindungen ${ }^{5)}$ mit Chlortrimethylsilan oder direkt aus den Halogenaromaten mit Hexamethyldisilan hergestellt ${ }^{6}$.

Da die getrennte Darstellung der metallorganischen Verbindungen häufig mit Nachteilen verbunden ist, z. B. erschwerte Metallierung sterisch gehinderter Positionen beim Arbeiten in niedrig siedenden Ethern (Grignard-Verbindungen) oder leicht erfolgende

*) Korrespondenz bitte an diesen Autor richten.

**) Herrn Prof. Dr. H. Pommer zum 60. Geburtstag gewidmet. 
Weiterreaktion (z. B. Isomerisierung oder Zersetzung) der Lithium- oder NatriumVerbindungen, lag es nahe, „in-situ“-Methoden") zur Gewinnung von Aryltrimethylsilanen anzuwenden. So wurden die Mehrfachsilylierung von Benzol ${ }^{8)}$ sowie die Silylierung einiger Alkyl-, Trimethylsiloxy- und Halogen-substituierter Benzole ${ }^{9,10)}$ durch direkte Umsetzung entsprechender Halogenaromaten mit Trimethylchlorsilan und Magnesium in einem geeigneten Lösungsmittel erfolgreich durchgeführt. Auch einige Heteroaryltrimethylsilane konnten auf diese Weise erhalten werden ${ }^{11)}$. Eine systematische Bearbeitung der ,in-situ“-Grignard-Synthese von Aryl- und Heteroaryltrimethylsilanen wurde jedoch noch nicht durchgeführt.

In der vorliegenden Arbeit berichten wir über die Darstellung von Aryl- und Heteroaryltrimethylsilanen nach dieser Methode, besonders bezüglich ihrer Anwendungsbreite, Grenzen und der optimalen Reaktionsbedingungen.

Zur Silylierung von Halogenaromaten wurden ,in-situ“-Grignard-Synthesen - mit Ausnahme der Polysilylierung von Halogenbenzolen ${ }^{8)}$ - bisher nur in Diethylether, Dibutylether oder Tetrahydrofuran durchgeführt. Diese Lösungsmittel können aber von Trimethylsilylhalogeniden gespalten werden ${ }^{12)}$, was besonders bei der Umsetzung sterisch gehinderter Verbindungen zur Bildung von Nebenprodukten führen kann. Als Lösungsmittel besonders geeignet erwies sich Hexamethylphosphorsäuretriamid (HMPT $)^{13)}$, da es einen hohen Siedepunkt hat und mit Wasser völlig mischbar ist. Zur Silylierung „in situ“ wurden Magnesium und Chlortrimethylsilan mit den Halogensubstituierten Aromaten im HMPT in der Regel $20-50 \mathrm{~h}$ bei $80^{\circ} \mathrm{C}$ umgesetzt; dann wurde hydrolytisch aufgearbeitet. Dieses „Eintopf"-Verfahren ermöglicht ohne getrennte Herstellung der Grignard-Zwischenstufe die Darstellung zahlreicher zum Teil noch nicht beschriebener Trimethylsilyl-substituierter Aromaten (Tabelle 1). Bromsubstituierte Aromaten reagieren hierbei rascher als Chlor-substituierte. In geringem Maße entstehen durch Reduktion auch die nicht silylierten Aromaten, die sich jedoch leicht destillativ abtrennen lassen.

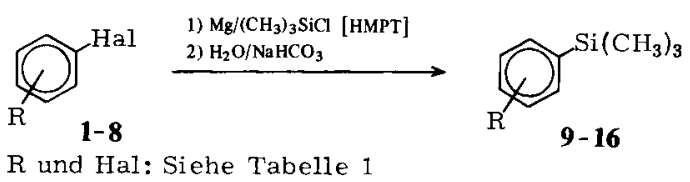

Die Überlegenheit der ,in-situ“-Methode bei Silylierungsreaktionen ist nicht nur in der einfacheren und bequemeren Durchführung der Reaktionen zu sehen, sondern auch in den in vielen Fällen besseren Ausbeuten (Tabelle 1).

Die aufgeführten Ergebnisse zeigen, daß sich die Halogenbenzole 1, Chlortoluole 3a-c und Bromxylole 3d,e mit sehr guten Ausbeuten (80-88\%) und die Dihalogenbenzole 2a,b mit 61- bzw. 67proz. Ausbeuten in die entsprechenden Aryltrimethylsilane überführen lassen. Außerdem gelingt die Silylierung sterisch gehinderter Arylhalogenide, wie Mesitylbromid (3f), Pentamethylphenylbromid (3q) und 2-tert-Butylphenylbromid (3h) sowie 2-Biphenylylchlorid (4c). Mit zunehmender sterischer Hinderung durch Substituenten sinken die Ausbeuten an Silylierungsprodukten. Eine Silylierung der sterisch besonders gehinderten 1-Brom-2,4,6-tri-tert-butyl- (3k) und 1-Brom-2,4,6tri-1-pyrrolidinylbenzole (8d) gelingt unter den Standardbedingungen nicht mehr; hier 


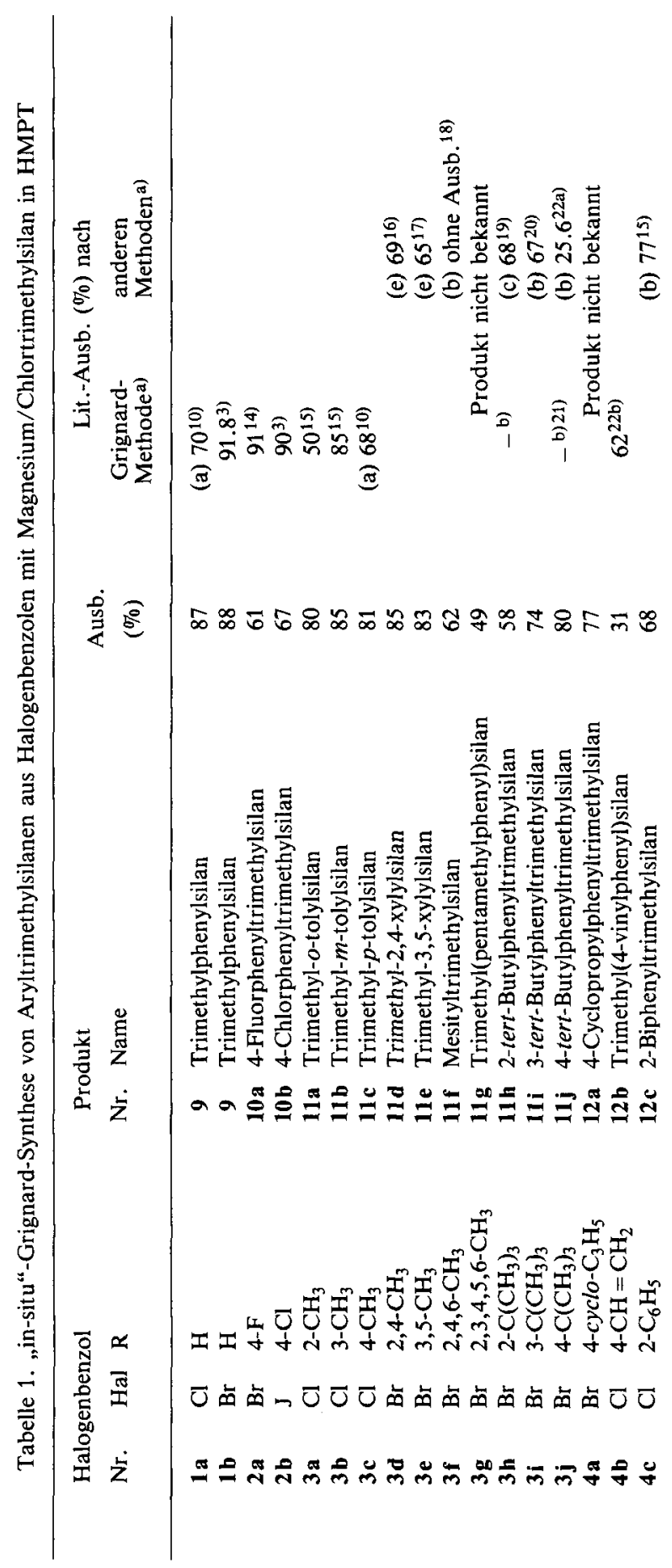




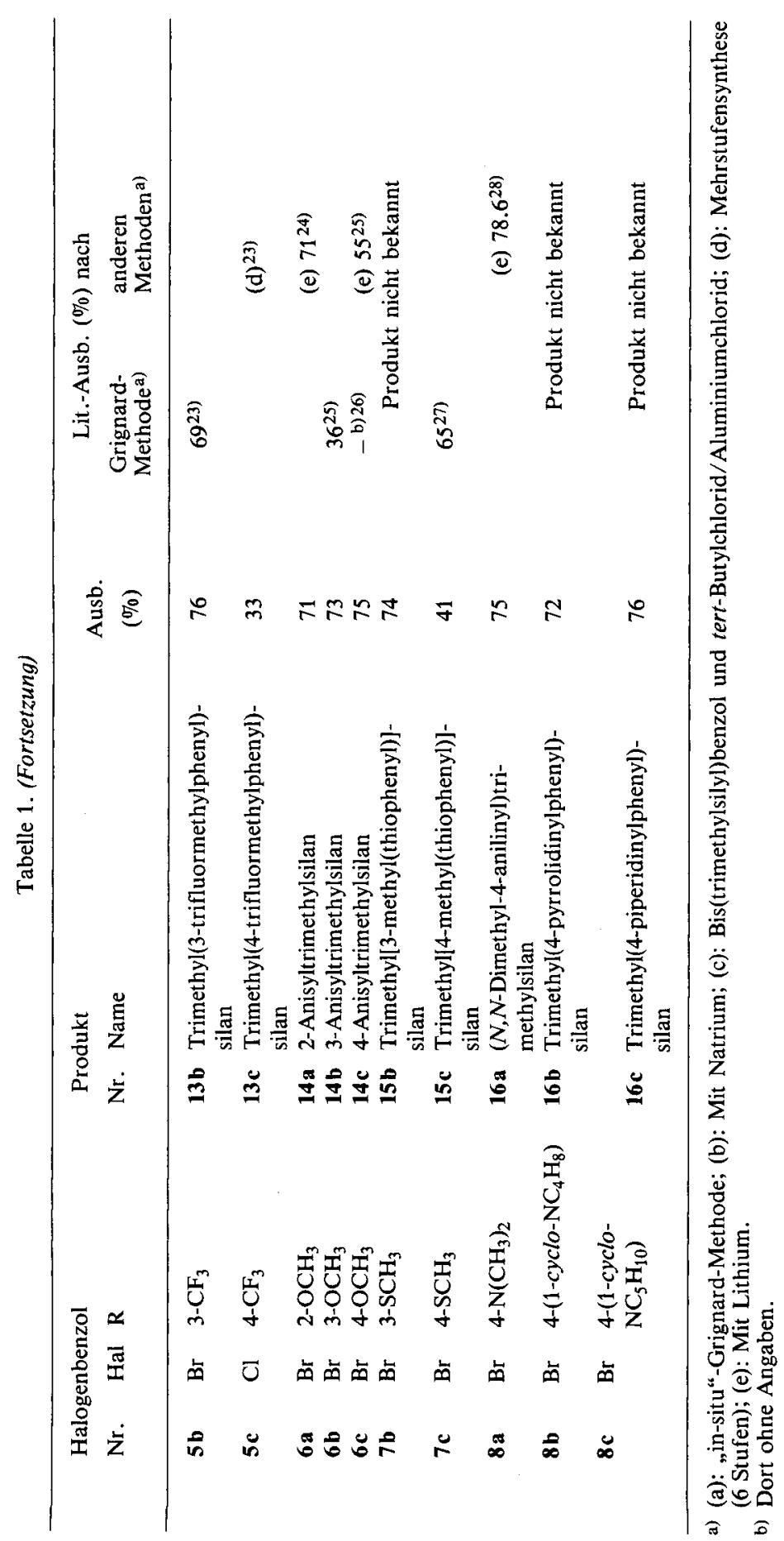


erfolgt lediglich Reduktion zu 1,3,5-Tri-tert-butyl- (17) bzw. 1,3,5-Tri-1-pyrrolidinylbenzol (18).<smiles>[R]c1cc([R])c(Br)c([R])c1</smiles>

$3 \mathbf{k}_{1} \mathbf{8 d}$
1) $\mathrm{Mg} /\left(\mathrm{CH}_{3}\right)_{3} \mathrm{SiCl}[\mathrm{HMPT}]$

2. $\mathrm{H}_{2} \mathrm{O} / \mathrm{NaHCO}_{3}$ oder $\mathrm{NaOH}$

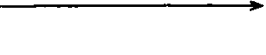

3k, 17: $\mathrm{R}=\mathrm{C}\left(\mathrm{CH}_{3}\right)_{3}$

8d, 18: $\mathrm{R}=\mathrm{N}_{-\mathrm{NH}_{2}-\mathrm{CH}_{2}}^{-\mathrm{CH}_{2}-\mathrm{CH}_{2}}$<smiles>[R]c1cc([R])cc([R])c1</smiles>

17,18

Auch das polymerisationsfreudige 4-Chlorstyrol (4b) kann in mäßiger Ausbeute nach der „in-situ“-Methode zum Trimethyl(4-vinylphenyl)silan (12b) umgesetzt werden.

Von den isomeren Trifluormethylphenyltrimethylsilanen 13 gelang uns die Darstellung der $m$ - und $p$-Isomeren $\mathbf{1 3 b}$ bzw. c, während das 2-Chlor(trifluormethyl)benzol (5a) unter den Reaktionsbedingungen eine Vielzahl von Produkten unaufgeklärter Struktur ergab. Auch die isomeren Anisylbromide 6 und deren $S$-Analoga 7 lassen sich ebenso wie die 4-Dialkylamino-substituierten Brombenzole 8a-c in guten Ausbeuten $(72-76 \%)$ nach der ,in-situ“-Grignard-Methode silylieren.

Zur Darstellung von Heteroaryltrimethylsilanen ist die „in-situ“-Grignard-Synthese ebenfalls geeignet, wie die Umsetzungen der isomeren Halogenpyridine 19 sowie des 2Bromthiophens (20) zeigen (Tabelle 2).

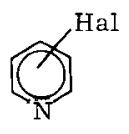

19a-d

Hal: Siehe Tabelle 2<smiles>Brc1cccs1</smiles>

20a

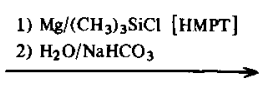

21a-d

Siehe Tabelle 2<smiles>CC(C)c1cccs1</smiles>

22a

Tabelle 2. „in-situ“-Grignard-Synthese von Heteroaryltrimethylsilanen aus Halogenheteroaromaten mit Magnesium/Chlortrimethylsilan in HMPT

\begin{tabular}{|c|c|c|c|c|c|c|}
\hline \multicolumn{2}{|c|}{ Halogenverb. } & \multicolumn{2}{|c|}{ Produkt } & \multirow{2}{*}{$\underset{(\%)}{\text { Ausb. }}$} & \multicolumn{2}{|c|}{$\begin{array}{l}\text { Lit.-Ausb. (\%) } \\
\text { nach Methode }\end{array}$} \\
\hline Nr. & $\mathrm{Hal}$ & Nr. & Name & & $\begin{array}{l}\text { "in-situ“- } \\
\text { Grignard }\end{array}$ & $\underset{\text { mit }}{\text { Lithium }}$ \\
\hline $19 \mathbf{a}$ & $2-\mathrm{Cl}$ & $21 \mathrm{a}$ & Trimethyl-2-pyridylsilan & 62 & $51^{11 a)}$ & \\
\hline $19 \mathrm{~b}$ & $3-\mathrm{Br}$ & $21 b$ & Trimethyl-3-pyridylsilan & 78 & & $24^{11 a)}$ \\
\hline $19 \mathrm{c}$ & $4-\mathrm{Br}$ & 21c & Trimethyl-4-pyridylsilan & 32 & & $39^{(1 a)}$ \\
\hline $19 d$ & $3,5-\mathrm{Br}_{2}$ & $21 d$ & 3,5-Bis(trimethylsilyl)pyridin & $\begin{array}{c}49 \\
\text { (roh) }\end{array}$ & Produkt $n$ & tt bekannt \\
\hline $20 \mathrm{a}$ & $2-\mathrm{Br}$ & $22 a$ & Trimethyl-2-thienylsilan & 78 & & 29) \\
\hline
\end{tabular}


Bei der Darstellung von Trimethyl-4-pyridylsilan (21c) polymerisiert ein Großteil des 4-Brompyridins (19c), was zu einer beträchtlichen Ausbeuteverminderung führt. Von den isomeren Bis(trimethylsilyl)pyridinen waren bisher die 2,5- und 2,6-Isomeren ${ }^{30 \text { ) }}$ bekannt; die Synthese des 3,5-Derivates 21d gelang aus 3,5-Dibrompyridin (19d) mit 2 Äquivalenten Chlortrimethylsilan und Magnesium; daneben entstanden das Mono(trimethylsilyl)-Derivat 21b (13\%) und in geringem Maße (ca. 5\%) das 3-Brom-5-(trimethylsilyl)pyridin (23), dessen Struktur durch Gaschromatographie kombiniert mit Massenspektrometrie (CI-MS) gesichert wurde.

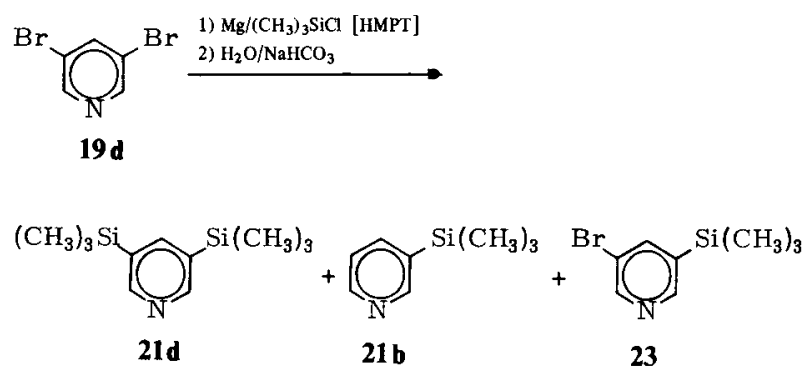

Das noch unbekannte Trimethyl-2-pyrimidinylsilan ist aus 2-Chlorpyrimidin unter den Bedingungen der ,in-situ“-Synthese nicht zugänglich. Aromatische Carbonyl-oder Cyan-substituierte Chlorverbindungen reagieren bei der „in-situ“-Synthese an der funktionellen Gruppe. Aus 4-Chloracetophenon (24) erhielten wir 2,3-Bis(4-chlorphenyl)-2,3-bis(trimethylsiloxy)butan (25), dessen Struktur ${ }^{1} \mathrm{H}$-NMR-spektroskopisch, durch Elementaranalyse und durch Molmassenbestimmung gesichert werden konnte. Acetophenon selbst reagiert unter ähnlichen Bedingungen ${ }^{31)}$.

2<smiles>C[C@@H](O)c1ccc(Cl)cc1</smiles>

24
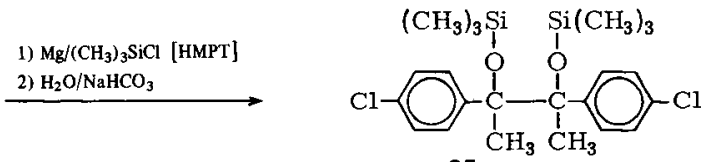

25

Aus 4-Chlorbenzonitril (26) entsteht in Gegenwart von Magnesium und Chlortrimethylsilan in mehrfachem Überschuß unter Silylierung und völliger Reduktion der funktionellen Gruppe das $N, N, \alpha, 4$-Tetrakis(trimethylsilyl)benzylamin (27), dessen solvolyseempfindliche $\mathrm{N}-\mathrm{Si}$-Bindungen mit ethanolischer Salzsäure gespalten werden können, wobei das Amin-hydrochlorid 28 entsteht. Das freie Amin wurde überraschenderweise auch nach der Umsetzung von Benzonitril mit Chlortrimethylsilan und Magnesium isoliert ${ }^{32)}$.
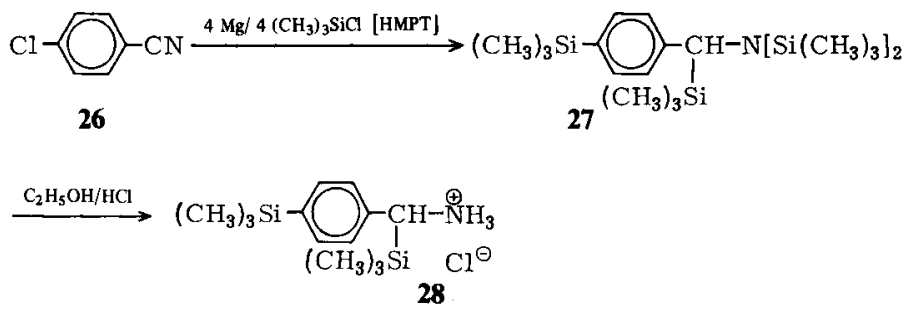
Arylhalogenide der beschriebenen Art verhalten sich also unter den Bedingungen der „in-situ“-Synthese wie ihre nicht halogenierten Stammverbindungen, deren Reaktionsweise mit Metallen, Chlortrimethylsilan und Donorsolventien von Calas und Mitarbeitern ${ }^{33)}$ kürzlich ausführlich zusammengefaßt worden ist.

Andere Arylhalogenide mit reduktionsempfindlichen funktionellen Gruppen, wie Nitrochlorbenzole, lassen sich nach der ,in-situ“-Methode erwartungsgemäß nicht mehr silylieren.

Trimethylnitrophenylsilane müssen daher durch Nitrierung von Trimethylphenylsilan ${ }^{34-36)}$, Nitrodesilylierung von Bis(trimethylsilyl)benzolen ${ }^{37,38)}$ oder durch Spaltung von Polysilanen mit Arylhalogeniden in Gegenwart von Katalysatoren ${ }^{39,40)}$ dargestellt werden.

Die Reaktion einer Reihe ,inerter" Aryl- und Heteroarylaromaten, wie z. B. 3Bromthiophen (20b), mit Magnesium unterliegt einer Hemmung ${ }^{41)}$. Wie erwartet, reagiert $20 \mathrm{~b}$ auch nach der ,in-situ“-Methode nicht. Bei Ausweitung der Methode auf andere Metalle erhielten wir jedoch mit 20b unter den Bedingungen einer modifizierten Wurtz-Fittig-Reaktion mit Natriumsand und Chlortrimethylsilan in Dioxan innerhalb von $13 \mathrm{~h}$ bei $60^{\circ} \mathrm{C}$ das völlig isomerenfreie Trimethyl-3-thienylsilan (22 b) in 80proz. Ausbeute. Dagegen ist z. B. das 3-Thienyllithium ${ }^{42)}$ nur bis $-70^{\circ} \mathrm{C}$ stabil und lagert sich bei höherer Temperatur in das 2-Isomere um.

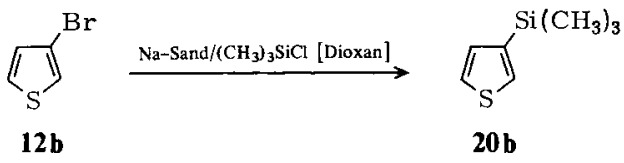

Auch mit dem weniger reaktiven System Zinkstaub/Chlortrimethylsilan in HMPT und Aryl- oder Heteroarylbromiden erhielten wir die entsprechenden Trimethylsilane; allerdings sind hierzu höhere Reaktionstemperaturen $\left(100-200^{\circ} \mathrm{C}\right)$ und noch längere Reaktionszeiten als bei den Umsetzungen mit Magnesium erforderlich. Arylchloride reagieren auf diese Weise nicht. - Zinkorganische Verbindungen wurden bisher nur zur aliphatischen $\mathrm{C}-\mathrm{Si}$-Bindungsknüpfung eingesetzt ${ }^{43)}$.

Dem Fonds der Chemischen Industrie danken wir für die Unterstützung dieser Arbeit, der Bayer $A G$ für die Überlassung von Chlortrimethylsilan.

\section{Experimenteller Teil}

Gaschromatographie (GC): Carlo-Erba-Fractovap GI mit FID, Säule $2 \mathrm{~m} \times 2 \mathrm{~mm}$ SE 30/3\% auf Chromosorb G. - ${ }^{1}$ H-NMR: Varian A 60 und EM 360; TMS interner oder externer Standard. - Kombination aus Gaschromatographie und Massenspektrometrie (GC/MS): CarloErba-Fractovap 2151-AC gekoppelt mit Finnigan 4023 mit Incos-Datensystem 2300.

„in-situ“-Grignard-Synthese von Aryl- und Heteroaryltrimethylsilanen. - Allgemeine Vorschrift für 0.2 molaren Ansatz: $\mathrm{Zu} 6.0 \mathrm{~g}(0.25 \mathrm{~mol})$ Magnesium , $120 \mathrm{ml}$ absol. HMPT und $27.16 \mathrm{~g}(0.25 \mathrm{~mol})$ Clortrimethylsilan läßt man einige Tropfen der Lösung von $0.2 \mathrm{~mol}$ Arylhalogenid in $50 \mathrm{ml}$ absol. HMPT (bei Feststoffen gesättigte Lösung, Gesamtmenge an HMPT jedoch konstant) tropfen. Man erwärmt, bis der Kolbeninhalt schäumt und startet die Reaktion durch Zugabe einiger Tropfen 1,2-Dibrommethan. Unter den in Tabelle 3 angegebenen Bedingungen wird der Rest der Arylhalogenid-Lösung unter Rühren zugetropft und anschließend gerührt. Beim Abkühlen fällt der Komplex $\mathrm{MgHa}_{2} \cdot 2 \mathrm{HMPT}$ aus. Der Kolbeninhalt wird unter 


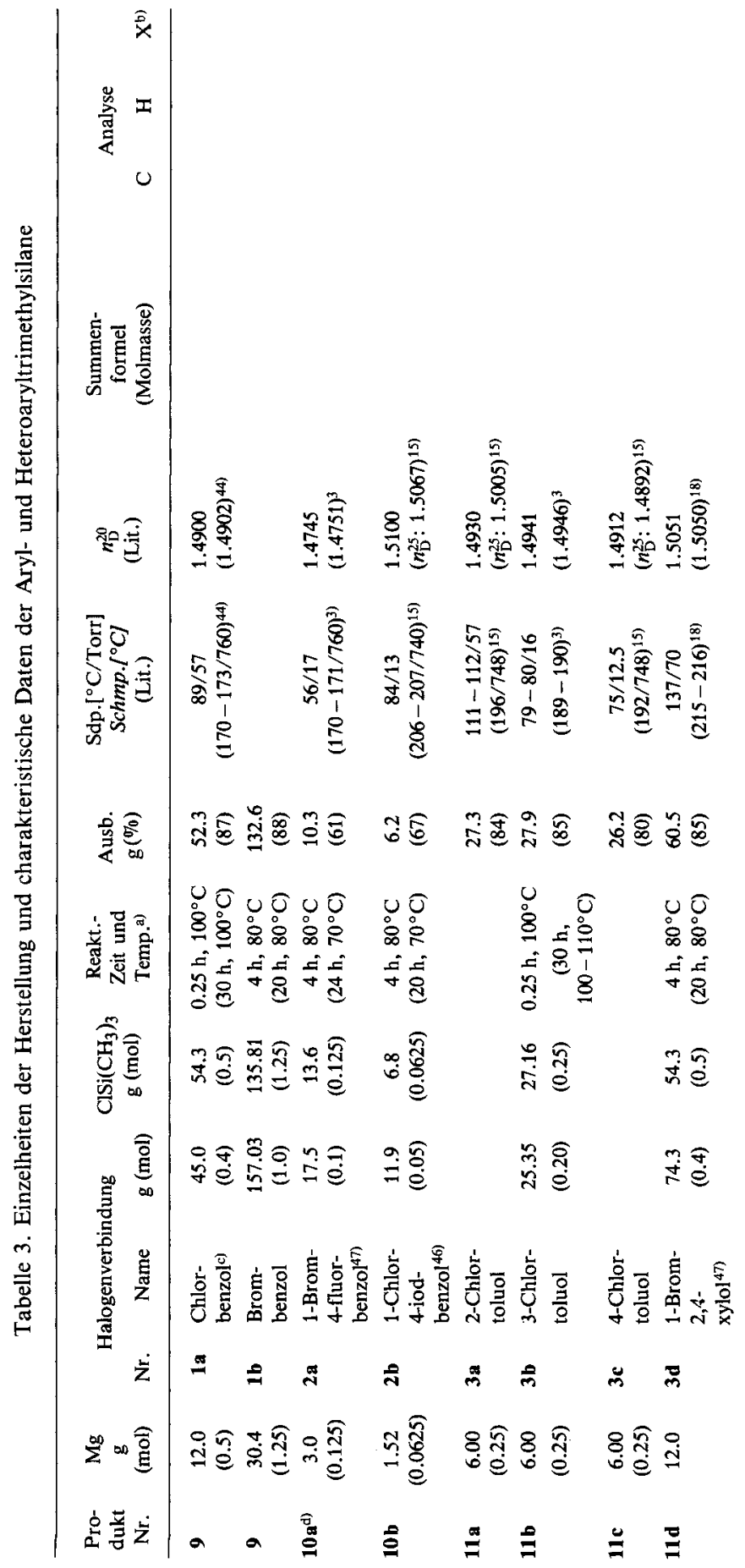




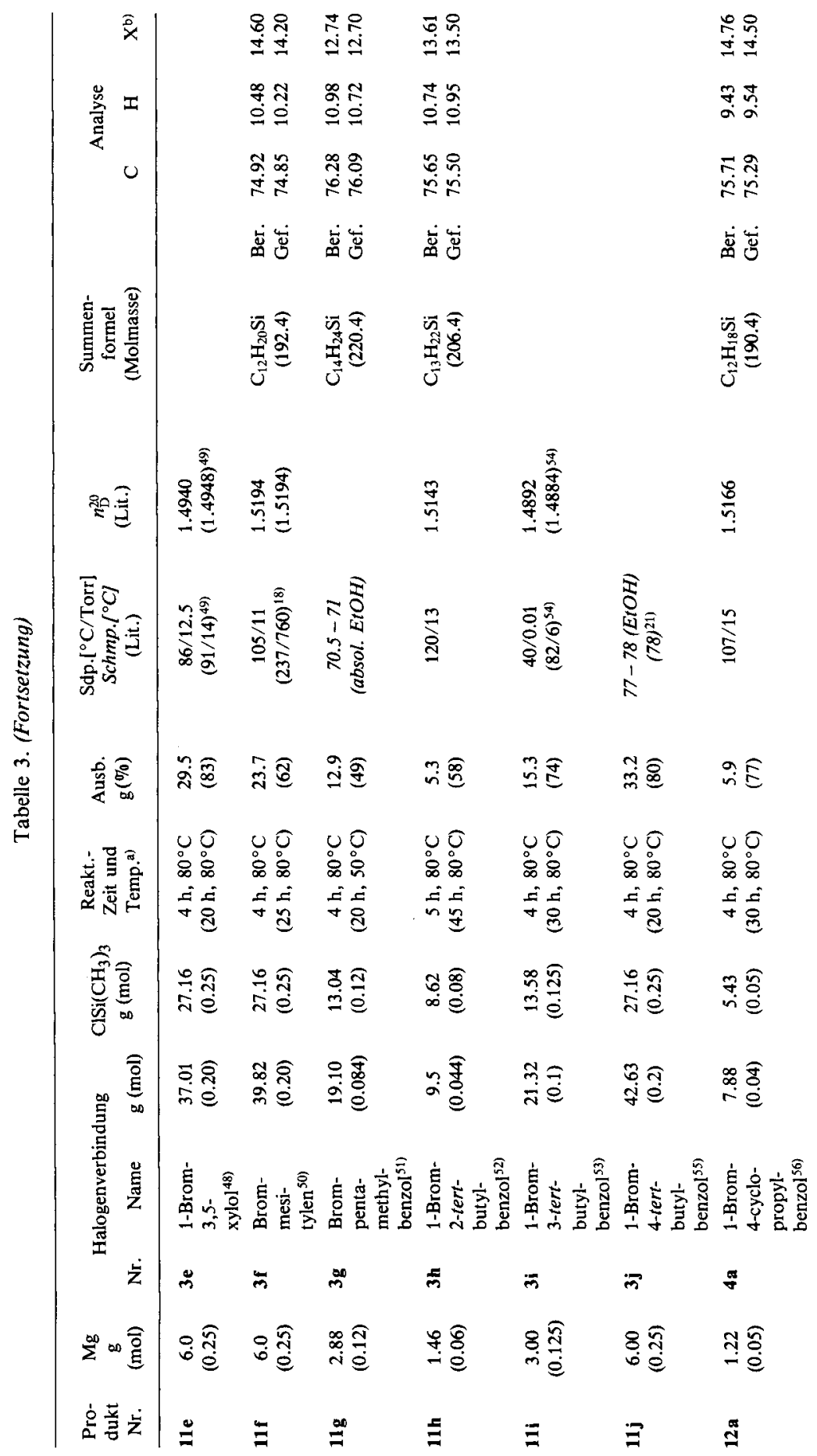




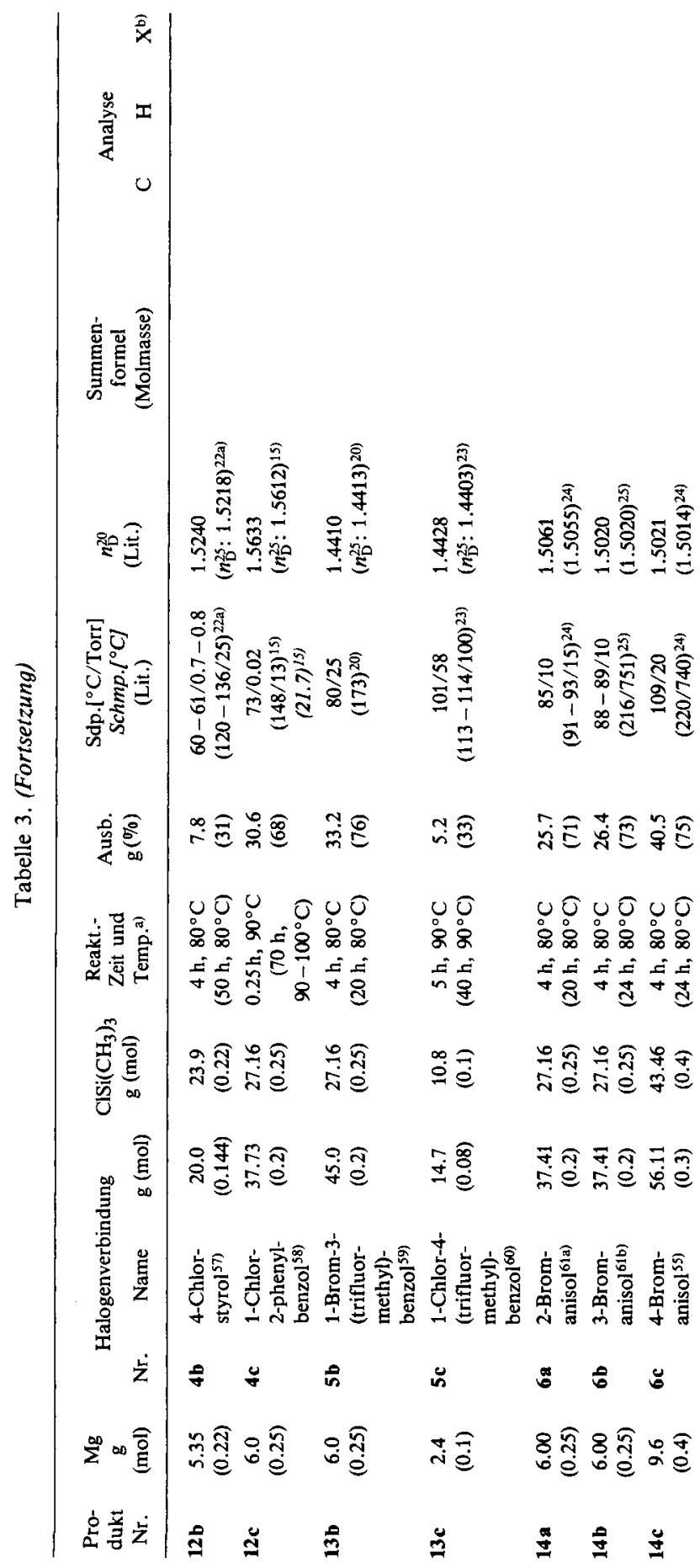




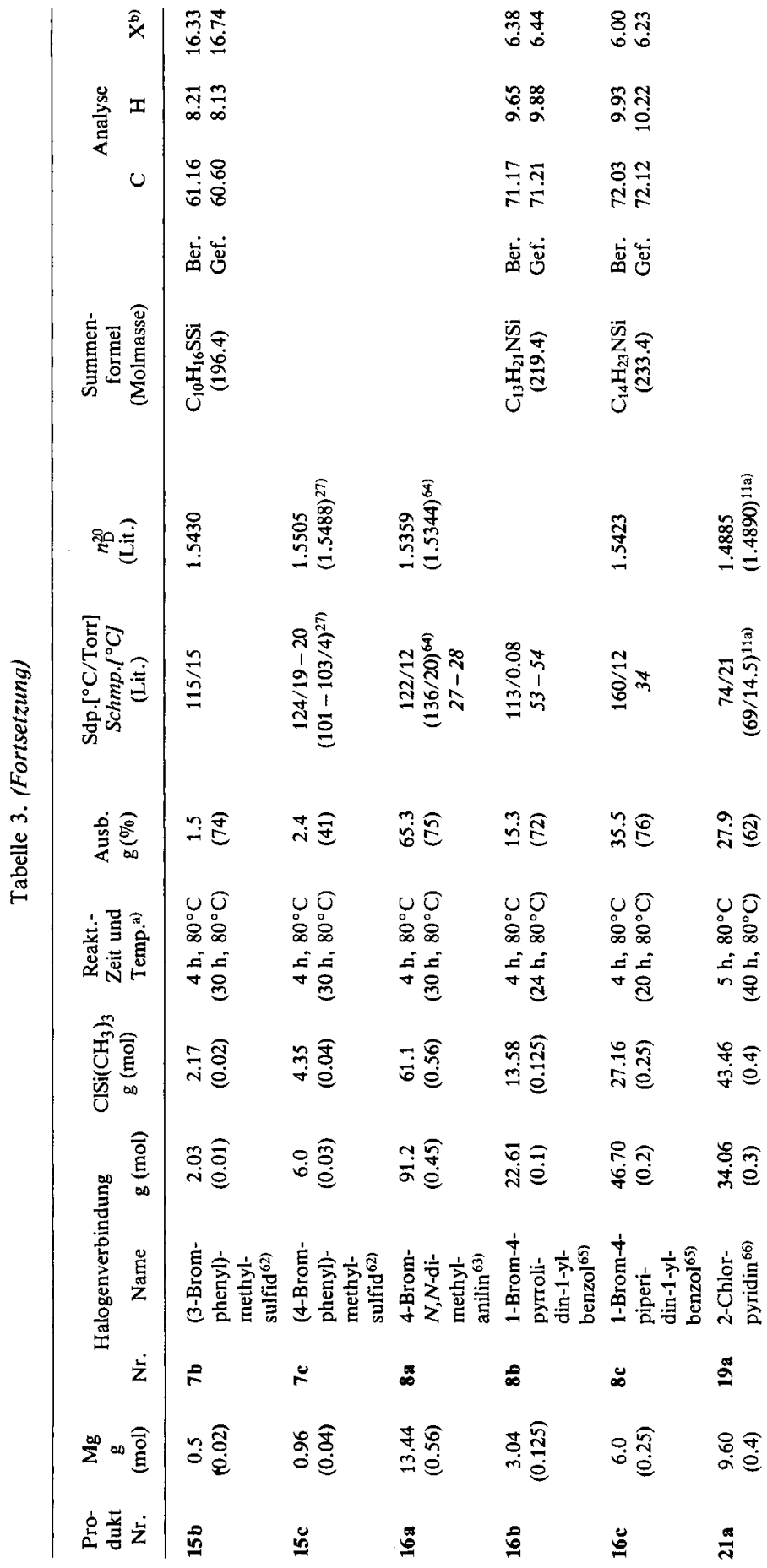




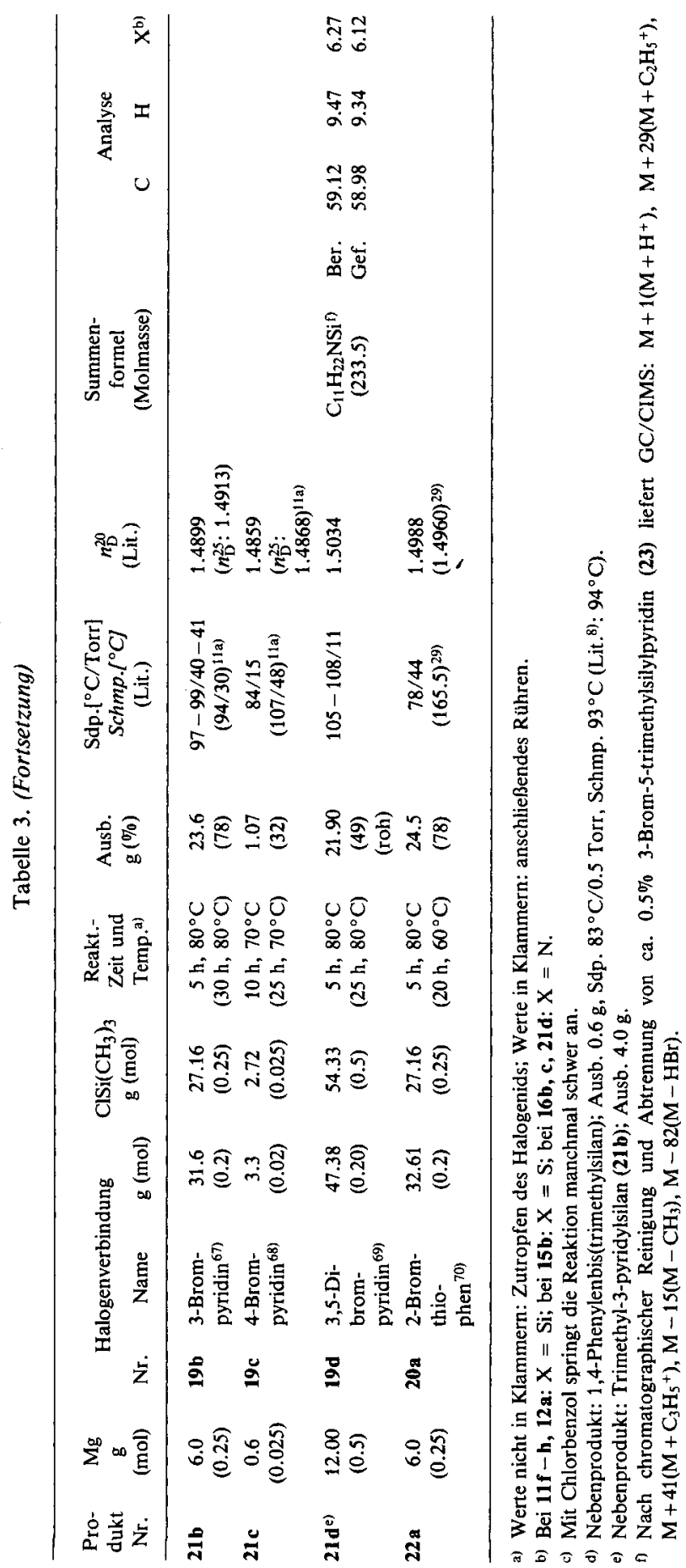


Rühren mit $1 \mathrm{l}$ eisgekühlter wäßriger Natriumhydrogencarbonat-Lösung (ca. $5 \mathrm{~g} / \mathrm{l}$ ) hydrolysiert, wobei auf Neutralität des Gemisches zu prüfen ist. Nach Absaugen wird das Silan-Derivat aus dem Filtrat mit Ether extrahiert, der Extrakt mit Wasser gewaschen, über Natriumsulfat getrocknet und nach Abdestillieren des Ethers der Rückstand fraktionierend destilliert oder umkristallisiert. Im Falle der Arylbromide können eventuell noch vorhandene Spuren Edukt durch kurzes Aufwärmen mit Natrium auf $60^{\circ} \mathrm{C}$ vor der Destillation entfernt werden. - Einzelheiten in den Tabellen 1, 2 und 3.

Umsetzung von 1-Brom-2,4,6-tri-tert-butylbenzol ${ }^{71)}(3 \mathbf{k})$ : In Modifizierung der allgemeinen Vorschrift (siehe oben) werden aus $1.46 \mathrm{~g}(0.06 \mathrm{~mol})$ Magnesium und $6.52 \mathrm{~g}(0.06 \mathrm{~mol})$ Chlortrimethylsilan in $100 \mathrm{ml} \mathrm{HMPT}$ nach $10 \mathrm{stdg}$. Zutropfen von $13.00 \mathrm{~g}(0.04 \mathrm{~mol}) 3 \mathrm{k}$ in $50 \mathrm{ml}$ HMPT bei $80^{\circ} \mathrm{C}$, 54stdg. Nachrühren bei $100^{\circ} \mathrm{C}$ und hydrolytischer Aufarbeitung $11.5 \mathrm{~g} \mathrm{kristallines} \mathrm{Ge-}$ misch aus 45\% 3k und 55\% 1,3,5-Tri-tert-butylbenzol (17) (durch GC bestimmt) gewonnen. Nach fraktionierender Kristallisation aus Ether erhält man als schwerlösliche Fraktion $2.3 \mathrm{~g}$ $(18 \%) 3 \mathrm{k}$ mit Schmp. $172^{\circ} \mathrm{C}$, (Lit. ${ }^{71)}: 173-174^{\circ} \mathrm{C}$ ) und als leicht lösliche Fraktion $1.1 \mathrm{~g}(11 \%)$ 17, mit Schmp. $72^{\circ} \mathrm{C}$ (Lit. ${ }^{72)}: 71-73^{\circ} \mathrm{C}$ ).

Umsetzung von 1-Brom-2,4,6-tri(1-pyrrolidinyl)benzol ${ }^{65)}$ (8d): In Modifizierung der allgemeinen Vorschrift (siehe oben) werden zu $1.22 \mathrm{~g}(0.05 \mathrm{~mol})$ Magnesium und $5.43 \mathrm{~g}(0.05 \mathrm{~mol})$ Chlortrimethylsilan in $100 \mathrm{ml} \mathrm{HMPT} \mathrm{in} 6 \mathrm{~h} 5.00 \mathrm{~g}(0.014 \mathrm{~mol}) 8 \mathrm{~d}$ in $70 \mathrm{ml} \mathrm{HMPT} \mathrm{bei} 80^{\circ} \mathrm{C}$ getropft; dann wird noch $20 \mathrm{~h}$ bei $80^{\circ} \mathrm{C}$ gerührt. Zur Hydrolyse wird die rote Lösung über einen Büchnertrichter direkt in $300 \mathrm{ml}$ stark gerührte eiskalte $0.2 \mathrm{~N}$ wäßrige Kaliumhydroxid-Lösung gegeben, der beigefarbene fein kristalline Niederschlag mit einer Glasfritte innerhalb 1 Woche abgesaugt, der feuchte Rückstand in $60 \mathrm{ml}$ Methanol gelöst, durch tropfenweise Zugabe von Wasser wieder ausgefällt, mit einer Glasfritte abgesaugt und im Exsikkator über Calciumchlorid getrocknet. Ausb. $3.7 \mathrm{~g}$ (93\%) 1,3,5-Tri(1-pyrrolidinyl)benzol (18); Schmp. $179-180^{\circ} \mathrm{C}$ (Lit. ${ }^{73)}$ : $179-181^{\circ} \mathrm{C}$ ).

Umsetzung von 4-Chloracetophenon ${ }^{74)}$ (24): In Modifizierung der allgemeinen Vorschrift (siehe oben) werden aus $6.00 \mathrm{~g}(0.25 \mathrm{~mol})$ Magnesium und $27.16 \mathrm{~g}(0.25 \mathrm{~mol})$ Chlortrimethylsilan in $100 \mathrm{ml}$ HMPT nach 20stdg. Zutropfen von $30.92 \mathrm{~g}(0.2 \mathrm{~mol}) 24$ in $50 \mathrm{ml} \mathrm{HMPT}$ bei $90^{\circ} \mathrm{C}, 40 \mathrm{stdg}$. Nachrühren bei $90^{\circ} \mathrm{C}$ und hydrolytischer Aufarbeitung $25 \mathrm{~g}$ gelbes Öl gewonnen, das fraktionierend destilliert wird. Ausb. $11.7 \mathrm{~g}$ hochviskoses Harz mit Sdp. $140^{\circ} \mathrm{C} / 0.01 \mathrm{Torr}$, das in absol. Ether kristallisiert. Ausb. $10.5 \mathrm{~g}(23 \%)$ farbloses kristallines 2,3-Bis(4-chlorphenyl)-2,3-bis(trimethylsiloxy)butan (25). - ${ }^{1} \mathrm{H} \cdot \mathrm{NMR}\left(\mathrm{CDCl}_{3} ; \mathrm{TMS}_{\text {ext. }}\right): \delta=-0.075\left[\mathrm{~s} ; 18 \mathrm{H}, \mathrm{Si}\left(\mathrm{CH}_{3}\right)_{3}\right], 1.37$ (s; $\left.6 \mathrm{H}, \mathrm{CH}_{3}\right), 7.35(\mathrm{~m} ; 8 \mathrm{H}$, Aromaten- $\mathrm{H})$.

$$
\begin{array}{cl}
\mathrm{C}_{22} \mathrm{H}_{32} \mathrm{O}_{2} \mathrm{Si}_{2} \text { (455.6) } & \text { Ber. C } 58.00 \mathrm{H} 7.08 \mathrm{Cl} 15.56 \text { Gef. C } 58.02 \mathrm{H} 6.87 \mathrm{Cl} 15.75 \\
& \text { Molmasse } 466 \text { (kryoskop. in Chloroform) }
\end{array}
$$

\section{Umsetzung von 4-Chlorbenzonitril ${ }^{75)}$ (26)}

1) In Modifizierung der allgemeinen Vorschrift (siehe oben) werden aus $6.00 \mathrm{~g}(0.25 \mathrm{~mol}) \mathrm{Ma}-$ gnesium und $27.16 \mathrm{~g}(0.25 \mathrm{~mol})$ Chlortrimethylsilan in $100 \mathrm{ml}$ HMPT nach Zutropfen von $11.0 \mathrm{~g}$ (0.08 mol) 26 in $50 \mathrm{ml} \mathrm{HMPT} \mathrm{in} 40 \mathrm{~h}$ bei $90^{\circ} \mathrm{C}$, 10stdg. Nachrühren bei $90^{\circ} \mathrm{C}$ sowie Vakuumdestillation $13.6 \mathrm{~g} \mathrm{(44 \% )} N, N, \alpha, 4$-Tetrakis(trimethylsilyl)benzylamin (27) mit Sdp. $122^{\circ} \mathrm{C}$ und $n_{\mathrm{D}}^{20}=1.5084$ gewonnen. $-{ }^{1} \mathrm{H}-\mathrm{NMR}\left(\mathrm{CDCl}_{3} ; \mathrm{TMS}_{\mathrm{ext} .}\right) \delta=0.16\left\langle\mathrm{~s} ; 18 \mathrm{H}, \mathrm{N}\left[\mathrm{Si}\left(\mathrm{CH}_{3}\right)_{3}\right]_{2}\right\rangle, 0.21$ [s; $\left.9 \mathrm{H}, \alpha-\mathrm{Si}\left(\mathrm{CH}_{3}\right)_{3}\right], 0.26\left[\mathrm{~s} ; 9 \mathrm{H}, 4-\mathrm{Si}\left(\mathrm{CH}_{3}\right)_{3}\right], 4.18(\mathrm{~s} ; 1 \mathrm{H}, \mathrm{CH}), 7.38(\mathrm{~m} ; 4 \mathrm{H}$, Aromaten-H).

$$
\begin{aligned}
& \mathrm{C}_{19} \mathrm{H}_{41} \mathrm{NSi}_{4} \text { (395.9) Ber. C } 57.64 \mathrm{H} 10.44 \mathrm{~N} 3.54 \text { Gef. C } 57.98 \text { H } 10.44 \text { N } 3.80 \\
& \text { Molmasse } 409 \text { (kryoskop. in Chloroform) }
\end{aligned}
$$

2) $1.95 \mathrm{~g} \mathrm{27}$, gelöst in $50 \mathrm{ml}$ warmem Ethanol, werden unter Rühren in $150 \mathrm{ml} 0.1 \mathrm{~N}$ Salzsäure getropft. Dann wird noch 10 min gerührt, der Niederschlag mit einer Glasfritte abgesaugt und aus Wasser umkristallisiert. Ausb. $1.42 \mathrm{~g}(99 \%) \alpha, 4$-Bis(trimethylsilyl)benzylammoniumchlorid (28) 
mit Schmp. $240.5-241^{\circ} \mathrm{C} .-{ }^{1} \mathrm{H}-\mathrm{NMR}\left(\mathrm{CDCl}_{3} ; \mathrm{TMS}_{\mathrm{ext}}\right) \delta=0.15\left[\mathrm{~s} ; 9 \mathrm{H}, \alpha-\mathrm{Si}\left(\mathrm{CH}_{3}\right)_{3}\right], 0.28$ [s; $\left.9 \mathrm{H}, 4-\mathrm{Si}\left(\mathrm{CH}_{3}\right)_{3}\right], 3.81(\mathrm{~s} ; 1 \mathrm{H}, \mathrm{CH}), 7.35\left(\mathrm{~s} ; 3 \mathrm{H}, \mathrm{NH}_{3}^{+}\right), 7.43(\mathrm{~m} ; 4 \mathrm{H}$, Aromaten-H).

$$
\begin{array}{llllll}
\mathrm{C}_{13} \mathrm{H}_{26} \mathrm{ClNSi}_{2} \text { (288.0) } & \text { Ber. C } 54.22 \text { H } 9.10 \mathrm{Cl} 12.31 \mathrm{~N} 4.86 \\
& \text { Gef. C } 54.17 \text { H } 9.19 \text { Cl } 12.27 \text { N } 4.86
\end{array}
$$

Tabelle 4. ${ }^{1} \mathrm{H}$-NMR-spektroskopische Daten neuer Aryl- und Heteroaryltrimethylsilane $\left(\mathrm{CDCl}_{3}\right.$;

\begin{tabular}{|c|c|c|}
\hline Nr. & \multicolumn{2}{|c|}{$\delta$-Werte (Multiplizität, Kopplungskonstanten, Zuordnung) } \\
\hline $11 \mathbf{f}$ & $0.38\left[\mathrm{~s} ; 9 \mathrm{H}, \mathrm{Si}\left(\mathrm{CH}_{3}\right)_{3}\right]$ & $\begin{array}{l}2.2\left(\mathrm{~s} ; 3 \mathrm{H}, 4-\mathrm{CH}_{3}\right), 2.38\left(\mathrm{~s} ; 6 \mathrm{H}, 2,6-\mathrm{CH}_{3}\right), 6.75(\mathrm{~s} ; 2 \mathrm{H}, \\
3,5-\mathrm{H})\end{array}$ \\
\hline $11 \mathrm{~g}$ & $0.41\left[\mathrm{~s} ; 9 \mathrm{H}, \mathrm{Si}\left(\mathrm{CH}_{3}\right)_{3}\right]$ & $\begin{array}{l}2.18\left(\mathrm{~s} ; 6 \mathrm{H}, 3,5-\mathrm{CH}_{3}\right), 2.21\left(\mathrm{~s} ; 3 \mathrm{H}, 4-\mathrm{CH}_{3}\right), 2.35(\mathrm{~s} ; 6 \mathrm{H}, \\
\left.2,6-\mathrm{CH}_{3}\right)\end{array}$ \\
\hline $11 \mathrm{~h}$ & $0.43\left[\mathrm{~s} ; 9 \mathrm{H}, \mathrm{Si}\left(\mathrm{CH}_{3}\right)_{3}\right]$ & $1.43\left[\mathrm{~s} ; 9 \mathrm{H}, \mathrm{C}\left(\mathrm{CH}_{3}\right)_{3}\right]$, ca. $7.5(\mathrm{~m} ; 4 \mathrm{H}$, Aromaten- $\mathrm{H})$ \\
\hline $12 \mathbf{a}$ & $0.27\left[\mathrm{~s} ; 9 \mathrm{H}, \mathrm{Si}\left(\mathrm{CH}_{3}\right)_{3}\right]$ & $\begin{array}{l}\text { ca. } 0.8\left[\mathrm{~m} ; 4 \mathrm{H},\left(\mathrm{CH}_{2}\right)_{2}\right], \text { ca. } 1.9(\mathrm{~m} ; 1 \mathrm{H}, \mathrm{CH}), 7.18(\mathrm{~d}, \mathrm{H} \\
J=8 \mathrm{~Hz} ; 2 \mathrm{H}, \text { Aromaten-H), } 7.50(\mathrm{~d}, J=8 \mathrm{~Hz} ; 2 \mathrm{H}, \\
\text { Aromaten-H) }\end{array}$ \\
\hline $15 b$ & $0.30\left[\mathrm{~s} ; 9 \mathrm{H}, \mathrm{Si}\left(\mathrm{CH}_{3}\right)_{3}\right]$ & $2.48\left(\mathrm{~s} ; 3 \mathrm{H}, \mathrm{SCH}_{3}\right)$, ca. $7.4(\mathrm{~m} ; 4 \mathrm{H}$, Aromaten-H) \\
\hline 16b & $0.24\left[\mathrm{~s} ; 9 \mathrm{H}, \mathrm{Si}\left(\mathrm{CH}_{3}\right)_{3}\right]$ & $\begin{array}{l}1.95\left(\mathrm{~m} ; 4 \mathrm{H}, \mathrm{CH}_{2}\right), 3.28\left(\mathrm{~m} ; 4 \mathrm{H}, \mathrm{NCH}_{2}\right), 6.62(\mathrm{~d}, J=8 \\
\mathrm{Hz} ; 2 \mathrm{H}, 3,5-\mathrm{N}), 7.45(\mathrm{~d}, J=8 \mathrm{~Hz} ; 2 \mathrm{H}, 2,6-\mathrm{H})\end{array}$ \\
\hline $16 \mathrm{c}$ & $0.23\left[\mathrm{~s} ; 9 \mathrm{H}, \mathrm{Si}\left(\mathrm{CH}_{3}\right)_{3}\right]$ & $\begin{array}{l}1.58\left(\mathrm{~m} ; 6 \mathrm{H}, \mathrm{CH}_{2}\right), 3.13\left(\mathrm{~m} ; 4 \mathrm{H}, \mathrm{NCH}_{2}\right), 6.85(\mathrm{~d}, J=8 \\
\mathrm{Hz} ; 2 \mathrm{H}, 3,5 \cdot \mathrm{H}), 7.36(\mathrm{~d}, J=8 \mathrm{~Hz} ; 2 \mathrm{H}, 2,6-\mathrm{H})\end{array}$ \\
\hline $21 \mathrm{~d}$ & $0.325\left[\mathrm{~s} ; 18 \mathrm{H}, \mathrm{Si}\left(\mathrm{CH}_{3}\right)_{3}\right]$ & $7.9(\mathrm{~m} ; 1 \mathrm{H}, 4-\mathrm{H}), 8.7(\mathrm{~m} ; 2 \mathrm{H}, 2,6-\mathrm{H})$ \\
\hline
\end{tabular}
TMS intern)

Umsetzung von 3-Bromthiophen ${ }^{76)}$ (18b) nach modifizierter Wurtz-Fittig-Reaktion: Mit einem Vibrator werden $4.12 \mathrm{~g}(0.18 \mathrm{~mol})$ Natrium in $80 \mathrm{ml}$ siedendem, über Calciumhydrid getrocknetem Dioxan zu Natriumsand verteilt. Nach Erkalten gibt man mit einer Spritze $10.86 \mathrm{~g}(0.1 \mathrm{~mol})$ frisch destilliertes Chlortrimethylsilan und dann bei $60^{\circ} \mathrm{C}$ unter starkem Rühren $8.15 \mathrm{~g}(0.05 \mathrm{~mol})$ $18 \mathrm{~b}$ in $20 \mathrm{ml}$ Dioxan innerhalb $1 \mathrm{~h}$ zu, rührt noch $12 \mathrm{~h}$ bei $60^{\circ} \mathrm{C}$, saugt über eine Glasfritte ab, spült mit $20 \mathrm{ml}$ getrocknetem Dioxan nach, destilliert aus dem Filtrat das überschüssige Chlortrimethylsilan und Dioxan bei Normaldruck ab und destilliert das zurückgebliebene Öl fraktionierend i. Vak. Ausb. $6.25 \mathrm{~g} \mathrm{(80 \% )} \mathrm{Trimethyl-3-thienylsilan} \mathrm{(20b)} \mathrm{mit} \mathrm{Sdp.} 88^{\circ} \mathrm{C} / 48.5$ Torr (Lit. ${ }^{77)}$ : $168^{\circ} \mathrm{C}$ ), $n_{\mathrm{D}}^{20}=1.4988$ (Lit. ${ }^{77)}: 1.4993$ ).

Umsetzungen mit Zinkorganischen Verbindungen. - Allgemeine Vorschrift: Zur Suspension von Zinkpulver in $50 \mathrm{ml}$ trockenem HMPT gibt man frisch destilliertes Chlortrimethylsilan, läßt unter Rühren innerhalb von 5 min die Lösung des Arylbromids in $25 \mathrm{ml} \mathrm{HMPT} \mathrm{tropfen,} \mathrm{rührt} \mathrm{un-}$ ter Erhitzen nach und arbeitet wie für die ,in-situ“-Methode (siehe oben) beschrieben auf.

Umsetzung von Brombenzol (1b): Aus $8.17 \mathrm{~g}(0.125 \mathrm{~mol})$ Zinkpulver, $13.58 \mathrm{~g}(0.125 \mathrm{~mol})$ Chlortrimethylsilan und $15.72 \mathrm{~g}(0.1 \mathrm{~mol}) 1 \mathrm{~b}$ erhält man nach $50 \mathrm{~h}$ bei $100^{\circ} \mathrm{C} 9.6 \mathrm{~g}(64 \%)$ Trimethylphenylsilan (9) mit Sdp. $65^{\circ} \mathrm{C} / 20$ Torr (Lit. ${ }^{44)}: 170-173^{\circ} \mathrm{C} / 760$ Torr).

Umsetzung von 3-Bromthiophen $^{76)}$ (20b): Aus $9.81 \mathrm{~g}(0.15 \mathrm{~mol})$ Zinkpulver, $16.29 \mathrm{~g}(0.15 \mathrm{~mol})$ Chlortrimethylsilan und $19.45 \mathrm{~g}(0.12 \mathrm{~mol}) 20 \mathrm{~b}$ erhält man nach $60 \mathrm{~h}$ bei $120^{\circ} \mathrm{C} 11.3 \mathrm{~g}(58 \%)$ eines Gemisches mit Sdp. $163-167^{\circ} \mathrm{C}$, das nach der gaschromatographischen und ${ }^{1} \mathrm{H}$-NMRspektroskopischen Untersuchung aus $75 \%$ Trimethyl-3-thienylsilan (22b) [Lit. ${ }^{77)}$ : Sdp. $168^{\circ} \mathrm{C}$ ] und 25\% Trimethyl-2-thienylsilan (22a) [Lit. ${ }^{77)}$ : Sdp. $165.5^{\circ} \mathrm{C}$ ] besteht. 
1) D. Häbich, Teil der Dissertation Univ. Stuttgart 1977.

2) 2a) C. Eaborn, J. Organomet. Chem. 100, 43 (1975); dort weitere Literaturhinweise. -2 b) $G$. Felix, J. Dunoguès, F. Piscolti und R. Calas, Angew. Chem. 89, 502 (1977); Angew. Chem., Int. Ed. Engl. 16, 488 (1977).

3) V. Chalousky und V. Bazant, Collect. Czech. Chem. Commun. 16, 580 (1951).

4) 4a) R. A. Benkeser, R. A. Hickner, D. I. Hoke und O. H. Thomas, J. Am. Chem. Soc. 80, 5289 (1958). - ${ }^{4 b)}$ D. Seyferth und S. C. Vick, J. Organomet. Chem. 141, 173 (1977).

5) C. Eaborn und R. A. Shaw, J. Chem. Soc. 1955, 1420.

6) M. A. Shippey und P. B. Dervan, J. Org. Chem. 42, 2654 (1977).

7) C. Blomberg und $F$. A. Hartog, Synthesis, 1977, 18.

8) P. Bourgeois, R. Calas, E. Jousseaume und J. Gerval, J. Organomet. Chem. 84, 165 (1975).

9) C. Eaborn, K. L. Jaura und D. R. M. Walton, J. Chem. Soc. 1964, 1198.

10) G. L. Larson, V. Nemeth und H. Valente, Synth. React. Inorg. Met.-Org. Chem. 6, 21 (1976).

11) 11a) D. G. Anderson, M. A. M. Bradney und D. E. Webster, J. Chem. Soc. B, 1968, 450. 11b) A. Fisher, M. W. Morgan und C. Eaborn, J. Organomet. Chem. 136, 323 (1977).

12) U. Krüerke, Chem. Ber. 95, 174 (1962).

13) H. Normant, Angew. Chem. 79, 1029 (1967); Angew. Chem., Int. Ed. Engl. 6, 1046 (1967).

14) J. D. Roberts, E. A. McElhill und R. Armstrong, J. Am. Chem. Soc. 71, 2915 (1949).

15) H. A. Clark, A. F. Gordon, C. W. Young und M. J. Hunter, J. Am. Chem. Soc. 73, 3798 (1951).

16) R. A. Benkeser. R. A. Hickner und D. I. Hoke, J. Am. Chem. Soc. 80, 5294 (1958).

17) R. A. Benkeser und H. R. Krysiak, J. Am. Chem. Soc. 76, 6353 (1954).

18) C. Eaborn und R. C. Moore, J. Chem. Soc. 1959, 3640.

19) C. Eaborn, D. R. M. Walton und D.J. Young, J. Chem. Soc. B, 1969, 15.

20) C. Eaborn und M. P. Jackson, J. Chem. Soc. B, 1969, 21.

21) C. Eaborn, J. Chem. Soc. 1956, 4858.

22) 22a) Yu. P. Egorov, L. A. Leites, N. G. Tolstikova und E. A. Chernyshev, Izv. Akad. Nauk SSSR, Otd. Khim. Nauk. 1961, 445 [Chem. Abstr. 55, 23402 (1961)]. - 22b) A. E. Senear, J. Wirth und R. G. Neville, J. Org. Chem. 25, 807 (1960).

23) C. W. Frost, J. Am. Chem. Soc. 78,3855 (1956).

24) H. Gilman und J. F. Nobis, J. Am. Chem. Soc. 72, 2629 (1956).

25) R. A. Benkeser und H. R. Krysiak, J. Am. Chem. Soc. 75, 4528 (1953).

26) C. Eaborn, J. Chem. Soc. 1953, 3148.

27) F. P. Bailey und R. Taylor, J. Chem. Soc. B, 1971, 1446.

28) H. Gilman, H. W. Melvin und J. J. Goodman, J. Am. Chem. Soc. 76, 3219 (1954).

29) C. Eaborn und J. A. Sperry, J. Chem. Soc. 1961, 4921.

30) E. Heilbronner, V. Hornung, F. H. Pinkerton und S. F. Thames, Helv. Chim. Acta, 55, 289 (1972).

31) R. Calas, J. Dunoguès, N. Duffaut und C. Biran, C. R. Acad. Sci., Ser. C, 267, 494 (1968).

32) C. Biran, R. Calas, J. Dunoguès und N. Duffaut, J. Organomet. Chem. 22, 557 (1970).

33) R. Calas und J. Dunoguès, J. Organomet. Chem. Libr. 2, 277 (1976).

34) J. L. Speier, J. Am. Chem. Soc. 75, 2930 (1953).

35) R. A. Benkeser und P. E. Blumfield, J. Am. Chem. Soc. 73, 4770 (1951).

36) Y. Limouzin und J. C. Maire, J. Organomet. Chem. 63, 51 (1973).

37) F. B. Deans und C. Eaborn, J. Chem. Soc. 1957, 498.

38) C. Eaborn, J. Organomet. Chem. 144, 271 (1978).

39) H. Matsumoto; S. Nagashima, K. Yoshihiro und Y. Nagai, J. Organomet. Chem. 85, 1 (1975).

40) D. Azarian, S. S. Dua, C. Eaborn und D. R. M. Walton, J. Organomet. Chem. 117, 55 (1976).

41) D. E. Pearson, D. Cowan und J. D. Beckler, J. Org. Chem. 24, 504 (1959).

42) S. Gronowitz, Ark. Kemi, 7, 361 (1954).

43) B. Gluck, C. Shaw und W. E. Smith, Brit. Pat. 601938 (14. Mai 1948) [Chem. Abstr. 42, 7317 (1948)].

44) H. C. Kaufinann, Handbook of Organometallic Compounds, 1. Aufl., S. 415, van Nostrand C., Princeton, New Jersey 1961.

45) $O$. Wallach und $F$. Heusler, Liebigs Ann. Chem. 243, 226 (1888).

46) M. Gomberg und L. H. Cone, Ber. Dtsch. Chem. Ges. 39, 3281 (1906).

47) J. B. Cohen und H. D. Dakin, J. Chem. Soc. 75, 893 (1899).

48) Hergestellt analog zu: H.R. Snyder, R. R. Adams und A. V. McIntosh, Jr., J. Am. Chem. Soc. 63, 3280 (1941). 
49) R. A. Benkeser und H. R. Krysiak, J. Am. Chem. Soc. 76, 6353 (1954).

50) A. H. Blatt, Organic Synthesis, 6. Aufl., Bd. 2, S. 95, Wiley and Sons, New York 1950.

51) A. Korczynski, Ber. Dtsch. Chem. Ges. 35, 871 (1902).

52) J. B. Shoesmith und A. Mackie, J. Chem. Soc. 1928, 2339.

53) J. I. G. Cadogan, D. H. Hey und G. H. Williams, J. Chem. Soc. 1954, 3352.

54) R. A. Benkeser, R. A. Hickner und D. I. Hoke, J. Am. Chem. Soc. 80, 2279 (1958).

55) Autorenkollektiv, Organikum, 15.Aufl., S. 394, VEB Deutscher Verlag der Wissenschaften, Berlin 1976.

56) R. Ya. Levina, P. A. Gembitskii und E. G. Treshchova, Zh. Obshch. Khim. 33, 371 (1963) [Chem. Abstr. 59, 7398 (1963)].

57) Organic Synthesis, Bd. 3, S. 204, Wiley and Sons, New York 1960.

58) H. Weingarten, J. Org, Chem. 26, 730 (1961).

59) J. H. Simons und E. O. Ramler, J. Am. Chem. Soc. 65, 389 (1943).

60) H. S. Booth, H. M. Elsey und P. E. Burchfield, J. Am. Chem. Soc. 57, 2066 (1935).

61) 61a) Durch Methylierung von o-Bromphenol: $R$. C. Huston und $A$. H. Neeley, J. Am. Chem. Soc. 57, 2176 (1935). - 61b) D. A. Shirley, Preparation of Organic Intermediates, 1. Aufl., S. 37, Wiley and Sons, New York 1951 .

62) V. Baliah und $M$. Uma, Tetrahedron, 19, 455 (1963).

63) A. Steinbach, Dissertation Univ. Stuttgart 1969.

64) R. A. Benkeser und H. R. Krysiak, J. Am. Chem. Soc. 75, 2421 (1953).

65) P. Menzel, Dissertation Univ. Stuttgart 1972.

66) H. v. Pechmann und O. Baltzer, Ber. Dtsch. Chem. Ges. 24, 3150 (1891).

67) H. Maier-Bode, Ber. Dtsch. Chem. Ges. 69, 1534 (1936).

68) J. P. Wibaut und F. W. Broekman, Rec. Trav. Chim. Pays-Bas, 58, 892 (1939).

69) E. E. Garcia, C. V. Greco und I. Moeyer-Hunsberger, J. Am. Chem. Soc. 82, 4430 (1960).

70) $\mathrm{Ng}$. Ph. Buu-Hoi, Liebigs Ann. Chem. 556, 1 (1944).

71) P. C. Myhre, Acta Chem. Scand. 14, 219 (1960).

72) P. C. Myhre, T. Rieger und J. T. Stone, J. Org. Chem. 31, 3425 (1966).

73) $F$. Effenberger und $R$. Niess, Chem. Ber. 101, 3787 (1968).

74) Siehe Lit. ${ }^{55)}$, dort S. 354.

75) Siehe Lit. ${ }^{55)}$, dort S. 591.

76) Organic Synthesis, Bd. 5, S. 149, Wiley and Sons, New York 1973.

77) F. B. Deans und C. Eaborn, J. Chem. Soc. 1959, 2303. 